\title{
MODELLING SPIN POLARIZED OPTOELECTRONIC PROCESSES
}

\author{
H. B. Sun $^{1}$ and M. C. de Oliveira ${ }^{1,2}$ \\ ${ }^{1}$ Department of Physics, The University of Queensland, QLD 4072, \\ Brisbane, Australia. \\ ${ }^{2}$ Departamento de Física, CCT, Universidade Federal de São Carlos, \\ 13565-905, São Carlos, SP, Brazil.
}

\begin{abstract}
We model the spin polarized optoelectronic processes in a GaAs-based light emitting diode under injection of spin-filtered electrons. To describe the microscopic dynamics we derive the quantum Langevin equations for photon number and carrier numbers. The polarization degree of the light generated by light emitting diode is calculated and compared with the experiment results.
\end{abstract}

\section{INTRODUCTION}

A new field of "spintronics" takes advantage of the spin of electrons and revolutionizes electronics leading to quantum devices. By understanding the behavior of electron spin in materials we can learn new fundamentals in solid-state physics. We study the quantum dynamics of spin-polarized optoelectronic processes in the system investigated experimentally $[1,2]$. The spin-polarised electrons are electrically injected into a GaAs-based light emitting diode. The applied magnetic field and the confinement lift the spin degeneracy and the light-hole and heavy-hole degeneracy respectively. The spin-polarised electrons transit between split conduction band and valence band by selection rules producing two types of circularly polarised light. electrons with spin $\pm 1 / 2$ in the conduction band recombine with holes of spin $\pm 3 / 2$ or $\mp 1 / 2$ in the valence band to emit photons in right $\left(\sigma^{+}\right)$or left $\left(\sigma^{-}\right)$circular polarization, respectively. The asymmetric transition probabilities causes a degree of polarisation of the light emitted from the device, which depends on the original degree of spin-polarisation of injected carriers and the amount of depolarisation caused by the scattering in the device. We use quantum Langevin equations $[3,4]$ to describe the quantum processes in such devices focusing on the active layer, and calculate the polarization degree of the emitted light.

\section{HAMILTONIAN AND QUANTUM LANGEVIN EQUATIONS}

The total Hamiltonian includes Hamiltonian of multi-mode photons, electrons and holes, interaction between carriers and photons, the many-body interaction among the carriers, leads, the interaction between the leads and the system, and the interaction between carriers and magnetic field:

$$
H=H_{c}+H_{p}+H_{d}+H_{m b}+H_{M}+H_{b a t h}+H_{b a t h-s y s} .
$$

Each term of the Hamiltonian is explained as follows. The carriers free Hamiltonian is

$$
H_{c}=\sum_{k}\left(\sum_{\mu} \varepsilon_{c k \mu} c_{k \mu}^{\dagger} c_{k \mu}+\sum_{\mu^{\prime}} \varepsilon_{v k \mu^{\prime}} d_{-k \mu^{\prime}}^{\dagger} d_{-k \mu^{\prime}}\right) .
$$

where $c_{k \mu}$ and $d_{-k \mu^{\prime}}$ are fermionic annihilation operators for electron with momentum $k$ and spin $\mu$ and holes with momentum $-k$ and spin $\mu^{\prime}$, respectively; $\varepsilon_{c k \mu}$ and $\varepsilon_{v k \mu^{\prime}}$ are the conduction and valence band energy, respectively. The Hamiltonian for multi-mode photons is given by 


$$
H_{p}=\sum_{l \mu \mu^{\prime}} \hbar \nu_{l} a_{l \mu \mu^{\prime}}^{\dagger} a_{l \mu \mu^{\prime}}
$$

with $a_{l \mu \mu^{\prime}}$ and $\nu_{l \mu \mu}^{\prime}$ the bosonic annihilation operator and frequency for photons in mode $l$ characterized by the allowed spin transition between $\mu$ and $\mu^{\prime}$. The dipole interaction is described by

$$
H_{d}=\sum_{l k \mu \mu^{\prime}} \hbar\left(g_{l k \mu \mu^{\prime}} d_{-k \mu^{\prime}}^{\dagger} c_{k \mu}^{\dagger} a_{l \mu \mu^{\prime}}+H . c .\right)
$$

where $g_{l k \mu \mu^{\prime}}$ is the dipole coupling constant (oscillator strength). The effect of the many body interaction $H_{m b}$ is included in the renormalized parameters of $\varepsilon_{c k \mu}, \varepsilon_{v k \mu^{\prime}}$ and $g_{l k \mu \mu^{\prime}}$ using a mean-field approximation [3]. The magnetic field Hamiltonian is given by

$$
H_{M}=\mu_{B} \mathbf{B} \cdot \sum_{k}\left(\sum_{\mu \nu} \mathcal{G}_{e} \mathbf{S}_{c \mu \nu} c_{k \mu}^{\dagger} c_{k \nu}+\sum_{\mu^{\prime} \nu^{\prime}} \mathcal{G}_{h} \mathbf{S}_{v \mu^{\prime} \nu^{\prime}} d_{-k \mu^{\prime}}^{\dagger} d_{-k \nu^{\prime}}\right)
$$

where $\mu_{B}$ is the Bohr magneton, $\mathcal{G}_{e(h)}$ is the electron (hole) Landé $g$-factor and $\mathbf{S}_{c}$ and $\mathbf{S}_{v}$ are spin matrices for electrons and holes, respectively. The reservoir Hamiltonian, $H_{\text {bath }}$ include the terms for photonic modes, electrons and holes and is conveniently eliminated in a Markovian approximation for the reduced dynamics of the device. The interaction with the carrier reservoir, $H_{b a t h-s y s}$ is considered in the Langevin approach, which include fluctuations in the carriers and photon populations.

When the magnetic field is orientated along the device (Faraday configuration), the Langevin equation for the dipole operator $\sigma_{k}^{\mu \mu^{\prime}}\left(=d_{-k \mu^{\prime}} c_{k \mu} e^{i \nu_{l} t}\right)$ and for the photon annihilation operator $A_{l \mu \mu^{\prime}}\left(=a_{l \mu \mu^{\prime}} e^{i \nu_{l} t}\right.$ ) describing the LED can be expressed as

$$
\begin{aligned}
\frac{d}{d t} \sigma_{k}^{\mu \mu^{\prime}}= & -\frac{i}{\hbar}\left(\varepsilon_{c k \mu}+\varepsilon_{v k \mu^{\prime}}-i \hbar \gamma-\hbar \nu_{l}\right) \sigma_{k}^{\mu \mu^{\prime}}-i \sum_{l} g_{l k \mu \mu^{\prime}}\left(1-n_{e k}^{\mu}-n_{h-k}^{\mu^{\prime}}\right) A_{l \mu \mu^{\prime}} \\
& -\frac{i}{\hbar} \mu_{B} B_{z}\left(\mathcal{G}_{e} S_{c \mu \mu}^{z} \sigma_{k}^{\mu \mu^{\prime}}+\mathcal{G}_{h} S_{v \mu^{\prime} \mu^{\prime}}^{z} \sigma_{k}^{\mu \mu^{\prime}}\right)+F_{\sigma k}^{\mu \mu^{\prime}} .
\end{aligned}
$$

and

$$
\frac{d}{d t} A_{l \mu \mu^{\prime}}=\left[-\frac{\kappa_{l}^{0}}{2}+i\left(\nu_{l}-\Omega_{l}\right)\right] A_{l \mu \mu^{\prime}}-i \sum_{k} g_{l k \mu \mu^{\prime}}^{*} \sigma_{k}^{\mu \mu^{\prime}}+F_{l} .
$$

where $\gamma$ and $\kappa_{l}^{0}$ are the rates of the dipole dephasing and the field decay respectively, $F_{\sigma k}^{\mu \mu^{\prime}}$ and $F_{l}$ are the fluctuation terms for the carriers and the field, respectively, and $\Omega_{l}$ is the passive-cavity frequency. In the slow varying regime for the adiabatic approximation, one gets the solution of Eq.(6)

$$
\sigma_{k}^{\mu \mu^{\prime}}=\frac{i \sum_{l^{\prime}} g_{l^{\prime} k \mu \mu^{\prime}}\left(n_{e k}^{\mu}+n_{h-k}^{\mu^{\prime}}-1\right) A_{l^{\prime} \mu \mu^{\prime}}+F_{\sigma k}^{\mu \mu^{\prime}}}{\left.\gamma+i\left[\mu_{B} B_{z}\left(\mathcal{G}_{e} S_{c \mu \mu}^{z}+\mathcal{G}_{h} S_{v \mu^{\prime} \mu^{\prime}}^{z}\right)+\varepsilon_{c k \mu}+\varepsilon_{v k \mu^{\prime}}-\hbar \nu_{l}\right)\right] / \hbar}
$$

Substituting it into Eq.(7), after a few steps of algebra calculation we obtain the photon number Langevin equation:

$$
\frac{d}{d t} n_{l \mu \mu^{\prime}}=-\kappa_{l}^{0} n_{l \mu \mu^{\prime}}+\sum_{l^{\prime}}\left(G_{l l^{\prime}}^{\mu \mu^{\prime}} A_{l \mu \mu^{\prime}}^{\dagger} A_{l^{\prime} \mu \mu^{\prime}}+H . c\right)+\left[\left(\sum_{\mu \mu^{\prime}} F_{\sigma l}^{\mu \mu^{\prime}}+F_{l}\right) A_{l \mu \mu^{\prime}}^{\dagger}+H . c .\right]
$$

where the polarized gain matrix, $G_{l l^{\prime}}^{\mu \mu^{\prime}}$ is defined as $G_{l l^{\prime}}^{\mu \mu^{\prime}}=\sum_{k} \mathcal{D}_{l k \mu \mu^{\prime}} g_{l k \mu \mu^{\prime}}^{*} g_{l^{\prime} k \mu \mu^{\prime}}\left(n_{e k}^{\mu}+n_{h-k}^{\mu^{\prime}}-1\right)$ and $F_{\sigma l}^{\mu \mu^{\prime}}=-i \sum_{k} g_{l k \mu \mu^{\prime}}^{*} \mathcal{D}_{l k \mu \mu^{\prime}} F_{\sigma k}^{\mu \mu^{\prime}}$ with

$$
\mathcal{D}_{l k \mu \mu^{\prime}}=\frac{1}{\left.\gamma+i\left[\mu_{B} B_{z}\left(\mathcal{G}_{e} S_{c \mu \mu}^{z}+\mathcal{G}_{h} S_{v \mu^{\prime} \mu^{\prime}}^{z}\right)+\varepsilon_{c k \mu}+\varepsilon_{v k \mu^{\prime}}-\hbar \nu_{l}\right)\right] / \hbar}
$$


We assume that the correlation between distinct modes can be neglected. Under the quasi-equilibrium condition

$$
\frac{d}{d t}\left\langle n_{e k}^{\mu}(t) n_{h-k}^{\mu^{\prime}}\right\rangle \ll 2 \gamma\left\langle n_{e k}^{\mu}(t) n_{h-k}^{\mu^{\prime}}\right\rangle
$$

we obtain the steady-state photon number in the model $l$ as

$$
\bar{n}_{l \mu \mu^{\prime}}=\frac{\kappa_{l}^{0} \bar{n}_{0}\left(\nu_{l}\right)+\left\langle R_{s p, l}^{\mu \mu^{\prime}}\right\rangle}{\kappa_{l}^{0}+\left(\left\langle R_{a b s, l}^{\mu \mu^{\prime}}\right\rangle-\left\langle R_{s p, l}^{\left.\mu \mu^{\prime}\right\rangle}\right\rangle\right)},
$$

where $R_{s p, l}^{\mu \mu^{\prime}}$ is the spontaneous emission rate into the mode $l$ due to the transition $\mu \mu^{\prime}$, and it reads

$$
R_{s p, l}^{\mu \mu^{\prime}} \equiv \frac{2}{\gamma} \sum_{k}\left|g_{l k \mu \mu^{\prime}}\right|^{2} \gamma^{2}\left|\mathcal{D}_{l k \mu \mu^{\prime}}\right|^{2} n_{e k}^{\mu} n_{h-k}^{\mu^{\prime}}
$$

and $R_{a b s, l}^{\mu \mu^{\prime}}$, the absorption rate is

$$
R_{a b s, l}^{\mu \mu^{\prime}} \equiv \frac{2}{\gamma} \sum_{k}\left|g_{l k \mu \mu^{\prime}}\right|^{2} \gamma^{2}\left|\mathcal{D}_{l k \mu \mu^{\prime}}\right|^{2}\left(1-n_{e k}^{\mu}\right)\left(1-n_{h-k}^{\mu^{\prime}}\right)
$$

The Eq.12 shows the influence of the absorption, emission rate and the number of thermal photons $\bar{n}_{0}\left(\nu_{l}\right)$ contributing to the photon numbers.

\section{POLARIZATION DEGREE OF THE EMITTED LIGHT}

In this section we study the measurement of the polarization degree light emitted from the LED. According to the input-output theory [5], the photon flux at the detector is given by $\bar{N}^{\mu \mu^{\prime}}=\beta_{0} \sum_{l}\left(\kappa_{l}^{0} \bar{n}_{0}\left(\nu_{l}\right)+R_{s p, l}^{\mu \mu^{\prime}}\right)$ where $\beta_{0}$ is the transference efficiency of the detection. The polarization degree of the detected light, $P \equiv\left(\bar{I}^{\sigma^{+}}-\bar{I}^{\sigma^{-}}\right) /\left(\bar{I}^{\sigma^{+}}+\bar{I}^{\sigma^{-}}\right)$, in the limit $\kappa_{l}^{0} \gg R_{a b s, l}^{\mu \mu^{\prime}}-R_{s p, l}^{\mu \mu^{\prime}}$, is calculated as

$$
P=\frac{\sum_{l}\left(R_{s p, l}^{-\frac{1}{2}-\frac{3}{2}}+R_{s p, l}^{\frac{1}{2}-\frac{1}{2}}-R_{s p, l}^{-\frac{1}{2} \frac{1}{2}}-R_{s p, l}^{\frac{1}{2} \frac{3}{2}}\right)}{\sum_{l}\left[\left(R_{s p, l}^{-\frac{1}{2}-\frac{3}{2}}+R_{s p, l}^{\frac{1}{2}-\frac{1}{2}}+R_{s p, l}^{-\frac{1}{2} \frac{1}{2}}+R_{s p, l}^{\frac{1}{2} \frac{3}{2}}\right)+4 \bar{n}_{0}\left(\nu_{l}\right)\right]}
$$

One can see the dependence of the polarization degree on the thermal photo number $\bar{n}_{0}\left(\nu_{l}\right)$ directly from the above equation while the effects of the magnetic field and the material dipole matrix for the polarization degree can be seen clearly trough the spontaneous emission rate, $R_{s p, l}^{\mu \mu^{\prime}}$ from Eq. (13). A preliminary numerical calculation is performed and the results are shown in Figure 1.
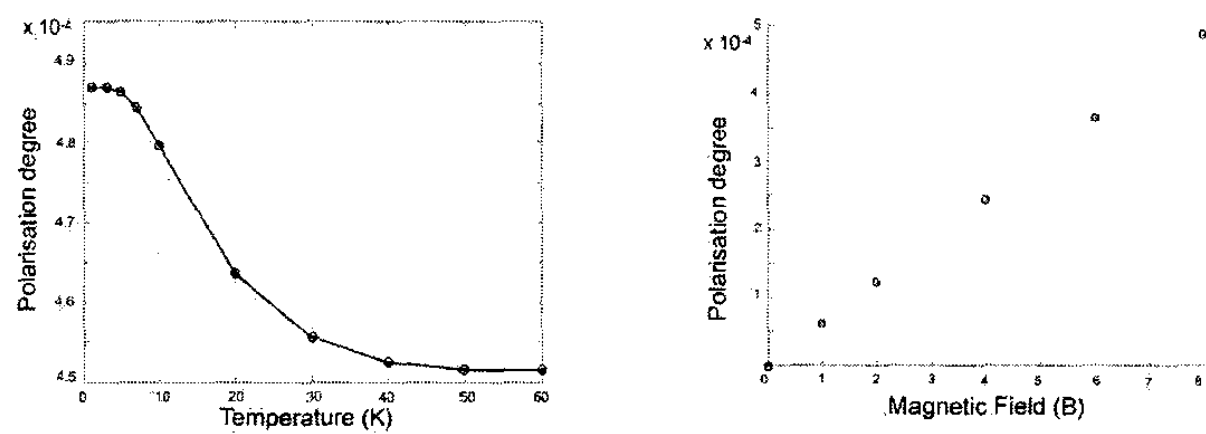

Figure 1: The degree of circular polarization of the electroluminescence in intrinsic GaAs: (a) Temperature dependence at $B=8 \mathrm{~T}$, (b) The magnetic field dependence at $\mathrm{T}=4.2 \mathrm{~K}$. 
Fig.1a presents the temperature dependence of the polarization degree which reflects not only the influence of the thermal photons but also the temperature dependence of carrier concentrations. The temperature dependence of the $\mathrm{g}$-factor is not included yet. Fig. $1 \mathrm{~b}$ shows the magnetic field dependence of the intrinsic polarization degree which causes the decline of the total degree of the circular polarization of the light from the LED as the spin splitting of GaAs is opposite in sign to that of the spin aligner $[2,6]$.

In a non-equilibrium case, effects of carrier pumping and recombination are important. We need a Langevin equation for the electron number operator including pumping, non-radiative recombination and dissipative effects as well. Non-radiative recombination will be introduced phenomenologically, following [4], where such a treatment shown to be successful in the treatment of low injection limit of carrier in $\mathrm{L}$ ED. The carrier number Langevin equation can be written as

$$
\frac{d}{d t} n_{e k}^{\mu}=\Lambda_{e k}^{\mu}\left(1-n_{e k}^{\mu}\right)-\gamma_{n r} n_{e k}^{\mu}+\sum_{l \mu^{\prime}}\left(i g_{l k \mu \mu^{\prime}} A_{l \mu \mu^{\prime}}^{\dagger} \sigma_{k}^{\mu \mu^{\prime}}+H . c .\right)+F_{e k}^{\mu}
$$

where $\Lambda_{e k}^{\mu}$ is the pumping rate due to a current injection, $\left(1-n_{e k}^{\mu}\right)$ is the pumping blocking, $\gamma_{n r}$ is the non-radiative recombination parameter, and $F_{e k}^{\mu}$ is the $\mu$-polarized electron number fluctuation term. Including these effects of pumping and non-radiative decay in the equation for the photon number and recalculating again the polarization degree give the degree of polarization as

$$
P=\frac{\sum_{k}\left\langle\Lambda_{e k}^{-1 / 2}\left(1-n_{e k}^{-1 / 2}\right)\right\rangle-\gamma_{n r}\left\langle n_{e}^{-1 / 2}\right\rangle-\sum_{k}\left\langle\Lambda_{e k}^{1 / 2}\left(1-n_{e k}^{1 / 2}\right)\right\rangle+\gamma_{n r}\left\langle n_{e}^{1 / 2}\right\rangle}{\sum_{k}\left\langle\Lambda_{e k}^{-1 / 2}\left(1-n_{e k}^{-1 / 2}\right)\right\rangle-\gamma_{n r}\left\langle n_{e}^{-1 / 2}\right\rangle+\sum_{k}\left\langle\Lambda_{e k}^{1 / 2}\left(1-n_{e k}^{1 / 2}\right)\right\rangle-\gamma_{n r}\left\langle n_{e}^{1 / 2}\right\rangle},
$$

which shows an explicit dependence with the electron pumping rate, for each spin component, and the non-radiative recombination of carriers, in which the electron-light-hole recombination is disregarded.

\section{SUMMARY}

We use the quantum Langevin approach to describe the dynamics in spin-polarized optoelectronic process and calculate the polarization degree of the emitted light from a LED due to injection of spin-polarized electron. The formalism for calculation including the spin polarized carrier pumping, non-radiative recombination has been established.

\section{REFERENCES}

(1) R. Fiederling, M. Kleim, G. Reuscher, W. Ossau, G. Schmidt, A. Waag, and L. W. Molenkamp, Nature 402, 787 (1999).

[2] B. T. Jonker, Y. D. Park, B. R. Bennett, H. D. Cheong, G. Kioseoglou, and A. Petrou, Phys. Rev. B 62, $8180(2000)$.

[3] W. W. Chow, S.W. Koch, and M. Sargent III, Semiconductor-Laser Physics (Spring-Verlag, Berlin, 1994).

[4] H. Fujisaki and A. Shimizu, Phys. Rev. A 57, 3074 (1998).

[5] D. F. Walls and G. J. Milburn, Quantum Optics, (Springer-Verlag, Berlin, 1995).

[6] G. Schmidt, L. W. Molenkamp, Physica E 9, 202 (2001). 